\title{
Introducing the "NewMed Effect":A New Phenomenon which Mimic Radiation Induced Bystander Effect and Amplifies the Biopositive Effects of Very Low Doses of Gamma Radiation
}

\author{
B. Nosrati ${ }^{1}$, SMJ Mortazavi ${ }^{2,3 *}$, T. Nejadsattari ${ }^{4}$ and P. Jonoubi ${ }^{5}$ \\ ${ }^{1}$ Department of Biology, Faculty of Sciences, Science and Research Branch, \\ Islamic Azad University , Tehran, Iran. \\ ${ }^{2}$ Ionizing and Non-ionizing Radiation Protection Research Center (INIRPRC), \\ Shiraz University of Medical Sciences, Shiraz, Iran. \\ ${ }^{3}$ Professor of Medical Physics, Medical Physics Department, School of Medicine, University of \\ Medical Sciences, Shiraz, Iran. \\ ${ }^{4}$ Department of Biology, Faculty of Sciences, Science and Research Branch, \\ Islamic Azad University , Tehran, Iran \\ ${ }^{5}$ Department of Plant Biology, Faculty of Biological Sciences Section, \\ Kharazmi University, Tehran, Iran.
}

http://dx.doi.org/10.13005/bbra/2124

(Received: 10 March 2016; accepted: 24 April 2016)

\begin{abstract}
In this paper we present our findings regarding a phenomenon which mimic bystander effect and significantly amplifies the stimulatory effects of low dose radiation. Thirty Triticum aestivum grains served as the "control group" while in the 2nd group 30 grains were exposed to gamma radiation emitted by Tc-99m. In the 3rd group, irradiated seeds were transferred to a new culture medium. The seeds in the 4th group were discarded after irradiation andt new un-irradiated cells were transferred to the irradiated medium. Exposure of Triticum aestivum to very low levels of ionizing radiation in the range of a few mSv enhanced root length, stem length, germination capacity, germination speed, fresh weight and the chlorophyll content. It was interestingly discovered that transferring irradiated seeds to a new medium, enhances root length, stem length, dry weight and the chlorophyll content. To the best of our knowledge this is the first report on the phenomenon we called it "NewMed effect". As far as we know, this is the 1st report on the stimulatory bio effects of exposure to very low doses of gamma radiation in Triticum aestivum. Further studies are needed to shed light on different aspects as well as potential applications of this effect.
\end{abstract}

Key words: Hormetic Effects, Low Dose Radiation,

Bystander Effect, Growth, Wheat, Triticum aestivum.

The bystander effect that refers to the effects in normal non-exposed cells adjacent to the irradiated or targeted cells (Hall 2003) has significantly challenged the concept that genetic and biochemical alterations are restricted only to the directly irradiated cells (Baskar 2010). Both

\footnotetext{
* To whom all correspondence should be addressed. E-mail:mmortazavi@sums.ac.ir
}

direct effects and bystander effects are dependent on factors such as the type of radiation and cell type (Baskar et al. 2007). Bystander effect that may either increase or decrease the radiation induced cancer risk has led to a major paradigm shift about the effects of ionizing radiation. The mechanisms of bystander effects are not yet clearly understood but it seems that this effect is induced by direct cell contact communications or release of specific materials from irradiated 
cells. It has been shown that bystander effect may cause stimulated cell growth or genotoxicity (intense double strand breaks (DSB), micronuclei (MN), mutation and decreased cell viability) in the non-irradiated bystander cells (Han et al. 2010). Some evidence indicate that in cultured cells, soluble factors released from the irradiated cells are responsible for bystander effects. For example, increased clonogenic stimulation in the bystander cells can be diminished by dilution of the culture medium of irradiated cells (Baskar et al. 2007; Ryan et al. 2008). When non-irradiated cells were co-cultured with cells exposed to low dose alpha particles with absorbed doses ranging 1-10 cGy, stimulated cell growth and increased MN and DSB were observed in the bystander cells (Han et al. 2010). Nitric oxide (NO) and transforming growth factor-1 (TGF-1) seem to play a role in increased cell proliferation in the non-irradiated bystander cells. On the other hand, increased proliferation (shortened cell cycle) in bystander cells does not let them have enough time to repair DSBs. Therefore, increased probability of mutation from the misrepaired or un-repaired DSBs can increase the risk of carcinogenesis in bystander cells (Han et al. 2010).

Over the past several years our laboratory has been focused on the health effects of exposure to elevated levels of natural ionizing radiation in high background natural radiation areas (HBNRAs) of Ramsar (Mortazavi et al. 2005a; Mortazavi et al. 2005b; Mortazavi and Karam 2005; Mortazavi and Mozdarani 2012; Mortazavi and Mozdarani 2013; Mortazavi et al. 2012b; Mortazavi et al. 2005c) and we have previously published the first report on the induction of adaptive response in the residents of these areas (Ghiassi-Nejad et al. 2002). A small part of Ramsar city with 1000-2000 population has high levels of natural radiation. The mean dose for the residents of HBNRAs of Ramsar is $10 \mathrm{mSv}$ y_1 but some of the residents receive doses as large as 260 mSv y_1 (Mortazavi and Mozdarani 2012). Altogether our findings showed no apparent harmful health effects and we suggested that global research on the residents of HBNRAs help scientists better justify if the old linear nothreshold model (LNT) of radiation risk is appropriate as the basis for public health measures in these areas (Mortazavi et al. 2005c).During our experiments, we realized that plants grown in the soil samples from HBNRAs of Ramsar showed increased germination, growth rate, wet weight and root length compared to those grown in ordinary soil. We also worked on phenomena which mimicked bystander effect by either transferring un-irradiated seeds to the irradiated culture medium, or transferring irradiated seeds to un-irradiated culture medium. In this paper our new findings regarding this phenomenon which mimic radiation induced bystander effect and significantly amplifies the stimulatory effects of low doses of ionizing radiation are discussed.

\section{MATERIALSANDMETHODS}

Wheat seeds were soaked in tap water for 12 hours at $25 \mathrm{C}$ in the dark. Then, their external surfaces were disinfected by sodium hypochlorite $5 \%$ ) and rinsed 3 times with double distilled water. A hatch of 30 grains served as the "control group" was placed on a sterile moist filter paper in a Petri dish and only sham exposed. In the 2nd group “Technetium group”, 30 grains, were exposed to gamma radiation emitted by Tc-99m at a distance of $20 \mathrm{~cm}$ from the radiation source. The exposure terminated after 24 hours. In the 3rd group, seeds received the exposure treatment of the 2nd group but after irradiation, the seeds were transferred to a new culture medium (distilled water). Finally, the seeds in the 4 th group again received the exposure treatment of the 2nd group but irradiated seeds were discarded and new un-irradiated cells were transferred to irradiated medium. The average dose received by the seeds during 24 hours of exposure was calculated by Monte Carlo (MC) simulation. The 3D geometry of MC simulation is shown in Figure 1. Figure 2 presents the $2 \mathrm{D}$ geometry of $\mathrm{MC}$ simulation showing the location of the cells in dose calculations. All Petri dishes were incubated at $22 \pm 1^{\circ} \mathrm{C}$ for 8 days.

To measure the seed germination potential, the daily count of the germinated seeds were carried out for eight days at specified time intervals. Seeds with root lengths of over two $\mathrm{mm}$ were considered as germinated (Melki 2010). Germination Capacity percentage (GC\%) was calculated according to the following equation (Melki 2008):

GC $(\%)=$ " Number of germinated seeds after 8 days" /"Total number of seeds" $\times 100$ 
All tests were repeated three times, and the results of root length stem length, germination capacity, germination speed, fresh weight, dry weight and the chlorophyll content were statistically analyzed by ANOVA test using SPSS software.

\section{RESULTS}

The mean $( \pm \mathrm{SD})$ dose received by the seeds during 24 hours of exposure as calculated by Monte Carlo (MC) simulation was $1105.4 \pm 378.8$ îSv (ranged 592.1 \pm 1939.3 ìSv).

\section{Root Length}

The mean root lengths in the control and exposure groups are shown in Figure 3. As it was expected, exposure of the seeds to low levels of gamma radiation has led to increased root length $(\mathrm{P}<0.001)$. Interestingly, when irradiated seeds were transferred to a new culture medium, the root length increased significantly again $(\mathrm{P}<0.05)$. On the other hand, when irradiated seeds were discarded and new seeds were transferred to the irradiated culture medium, the mean root length in this group was not significantly different from that of the control group ( $\mathrm{P}=0.98$ ).

\section{Stem Length}

Figure 4 shows the mean stem lengths in the control and exposure groups. Again as it was expected, exposure to low levels of gamma radiation significantly increased the stem length $(\mathrm{P}<0.001)$. In contrast with root length findings, when irradiated seeds were transferred to a new culture medium, the stem length did not increased

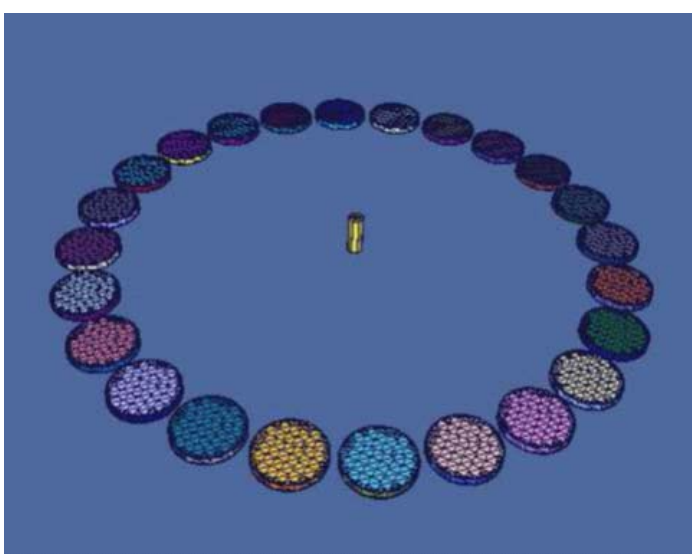

Fig. 1. The 3D geometry of MC simulation significantly $(\mathrm{P}=0.78)$. Showing a similar pattern of results, when irradiated seeds were discarded and new seeds were transferred to the irradiated culture medium, the mean stem length in this group was not significantly different from that of the control group $(\mathrm{P}=0.82)$.

\section{Fresh Weight}

The mean fresh weights in the control and exposure groups are shown in Figure 5. Again showing a similar pattern of results, exposure to low levels of gamma radiation has led to increased fresh weight $(\mathrm{P}<0.001)$. Again, in contrast with root length findings, when irradiated seeds were transferred to a new culture medium, the fresh weight did not increased significantly $(\mathrm{P}=0.31)$. Again, when irradiated seeds were discarded and new seeds were transferred to the irradiated culture medium, the mean fresh weight in this group was not significantly different from that of the control group $(\mathrm{P}=0.22)$.

\section{Dry Weight}

The mean dry weights in the control and exposure groups are shown in Figure 6. In contrast with our previous findings, exposure to low levels of gamma radiation did not significantly increase the dry weight $(\mathrm{P}=0.40)$. Interestingly, in spite of this, when irradiated seeds were transferred to a new culture medium, the dry weight increased significantly $(\mathrm{P}<0.001)$. In contrast with our previous findings, when irradiated seeds were discarded and new seeds were transferred to the irradiated culture medium, the mean dry weight in this group was significantly lower than that of the control group $(\mathrm{P}<0.001)$.

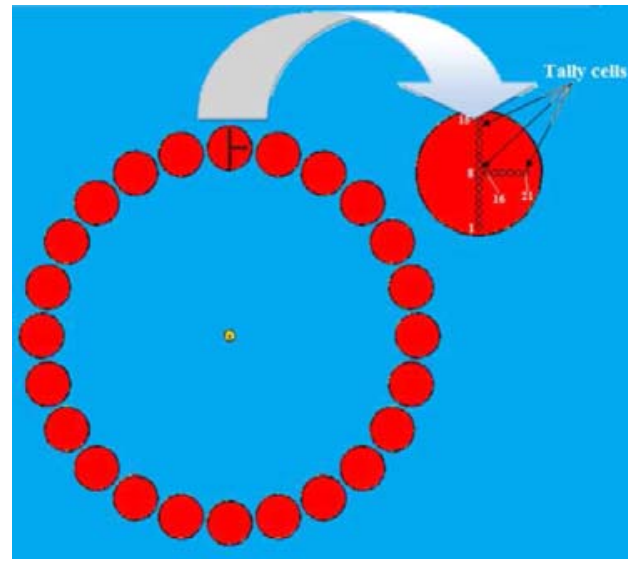

Fig. 2. The 2D geometry of MC simulation which shows the location of the cells in dose calculation 


\section{Germination Capacity}

Figure 7 shows the mean germination capacity in the control and exposure groups. Again as it was expected, exposure to low levels of gamma radiation significantly increased the germination capacity $(\mathrm{P}<0.001)$. In contrast with root length findings, when irradiated seeds were transferred to a new culture medium, the germination capacity did not increased significantly $(\mathrm{P}=0.99)$. Showing

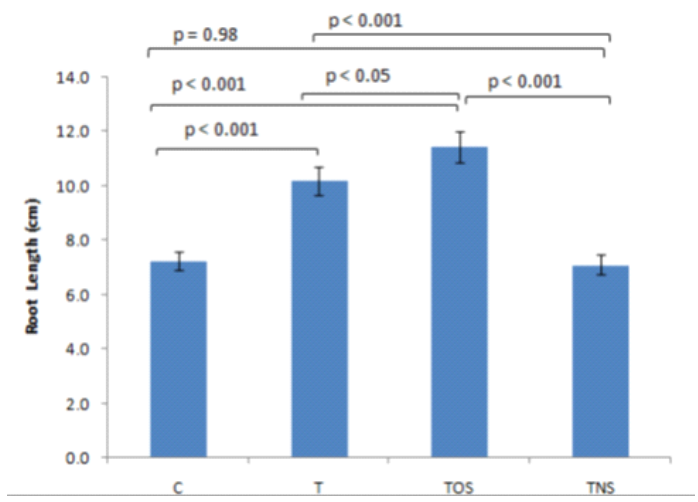

T: Seeds exposed to gamma rays from a Tc-99m source TOS: The same exposure condition but irradiated seeds were transferred to a new culture medium.

TNS: The same exposure condition but new un-irradiated seeds were transferred to the irradiated culture medium Error Bars indicate standard deviation (SD).

Fig. 3. The mean root length in the control and exposure groups

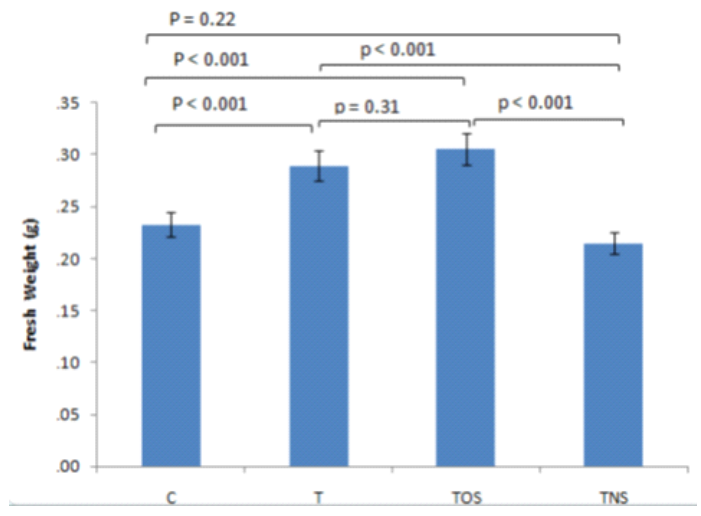

T: Seeds exposed to gamma rays from a Tc-99m source TOS: The same exposure condition but irradiated seeds were transferred to a new culture medium.

TNS: The same exposure condition but new un-irradiated seeds were transferred to the irradiated culture medium Error Bars indicate standard deviation (SD).

Fig. 5. The mean fresh weight in the control and exposure groups a similar pattern of results, when irradiated seeds were discarded and new seeds were transferred to the irradiated culture medium, the mean germination capacity in this group was significantly lower than that of the control group $(\mathrm{P}<0.05)$.

\section{Germination Speed}

The mean germination speeds in the control and exposure groups are shown in Figure 8. Again, exposure to low levels of gamma radiation

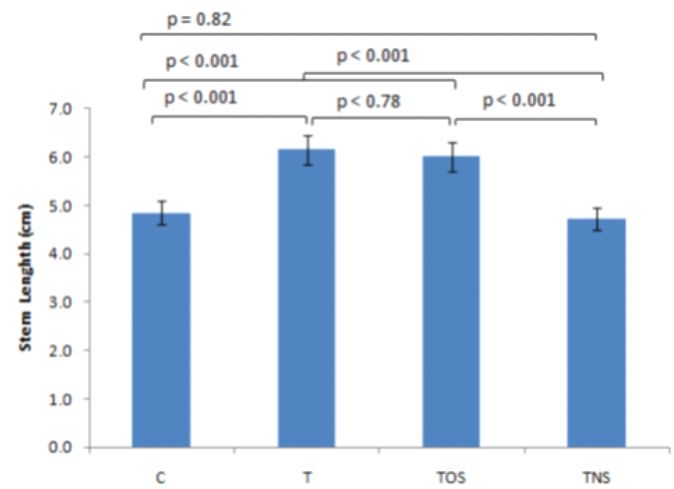

T: Seeds exposed to gamma rays from a Tc-99m source TOS: The same exposure condition but irradiated seeds were transferred to a new culture medium.

TNS: The same exposure condition but new un-irradiated seeds were transferred to the irradiated culture medium Error Bars indicate standard deviation (SD).

Fig. 4. The mean stem length in the control and exposure groups

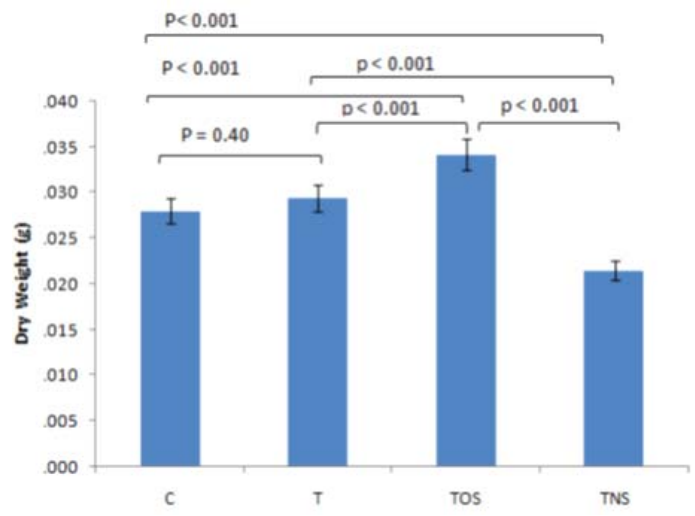

T: Seeds exposed to gamma rays from a Tc-99m source TOS: The same exposure condition but irradiated seeds were transferred to a new culture medium.

TNS: The same exposure condition but new un-irradiated seeds were transferred to the irradiated culture medium Error Bars indicate standard deviation (SD).

Fig. 6. The mean dry weight in the control and exposure groups 
significantly increased fresh weight $(\mathrm{P}<0.001)$. Again, in contrast with root length findings, when irradiated seeds were transferred to a new culture medium, the fresh weight did not increased significantly ( $\mathrm{P}=0.99)$. Again, when irradiated seeds were discarded and new seeds were transferred to the irradiated culture medium, the mean fresh weight in this group was not significantly different from that of the control group $(\mathrm{P}=0.99)$.

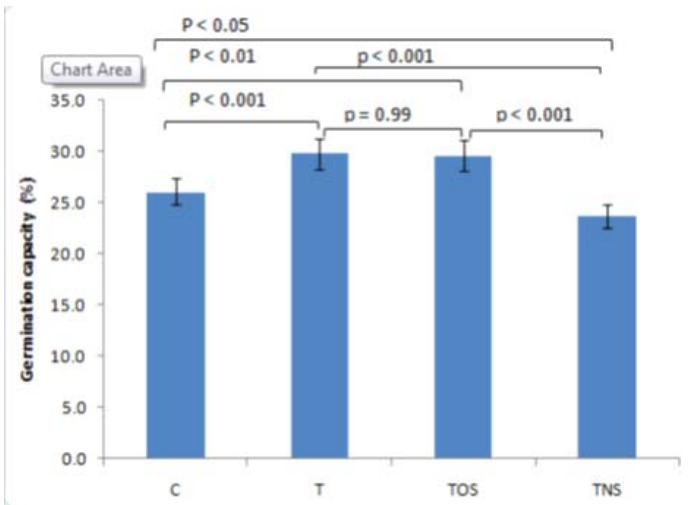

T: Seeds exposed to gamma rays from a Tc-99m source TOS: The same exposure condition but irradiated seeds were transferred to a new culture medium.

TNS: The same exposure condition but new un-irradiated seeds were transferred to the irradiated culture medium Error Bars indicate standard deviation (SD).

Fig. 7. The mean germination capacity in the control and exposure groups

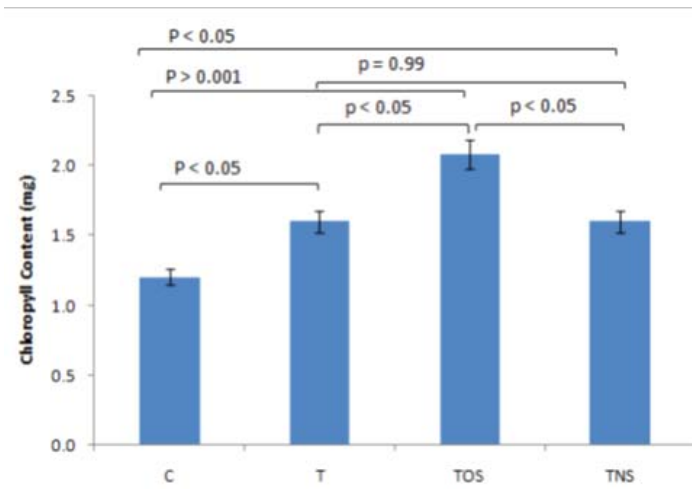

T: Seeds exposed to gamma rays from a Tc-99m source TOS: The same exposure condition but irradiated seeds were transferred to a new culture medium.

TNS: The same exposure condition but new un-irradiated seeds were transferred to the irradiated culture medium Error Bars indicate standard deviation (SD).

Fig. 9. The mean chlorophyll content in the control and exposure groups

\section{Chlorophyll Content}

Figure 9 shows the mean chlorophyll contents in the control and exposure groups. Again as it was expected, exposure to low levels of gamma radiation significantly increased the germination capacity $(\mathrm{P}<0.05)$. In a similar pattern with our previous results about root length, when irradiated seeds were transferred to a new culture medium, the germination capacity significantly increased

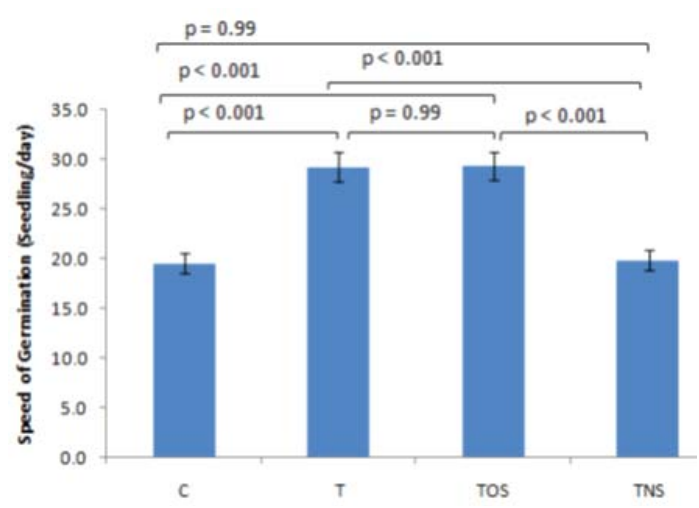

T: Seeds exposed to gamma rays from a Tc-99m source TOS: The same exposure condition but irradiated seeds were transferred to a new culture medium.

TNS: The same exposure condition but new un-irradiated seeds were transferred to the irradiated culture medium Error Bars indicate standard deviation (SD).

Fig. 8. The mean germination speed in the control and exposure groups

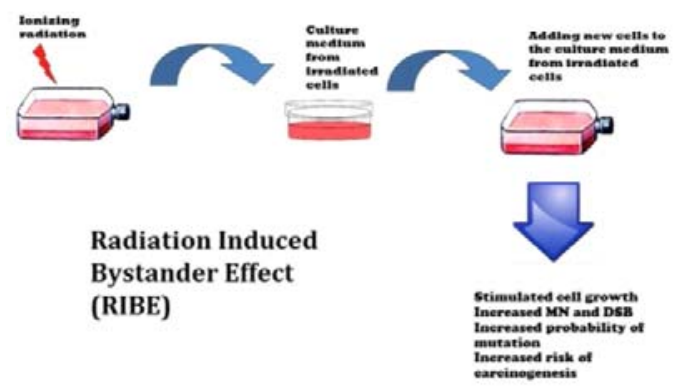

Fig. 10. Graphical representation of the radiation induced bystander effect

$(\mathrm{P}<0.05)$. On the other hand, when irradiated seeds were discarded and new seeds were transferred to the irradiated culture medium, the mean germination capacity in this group was significantly higher than that of the control group $(\mathrm{P}<0.05)$. 


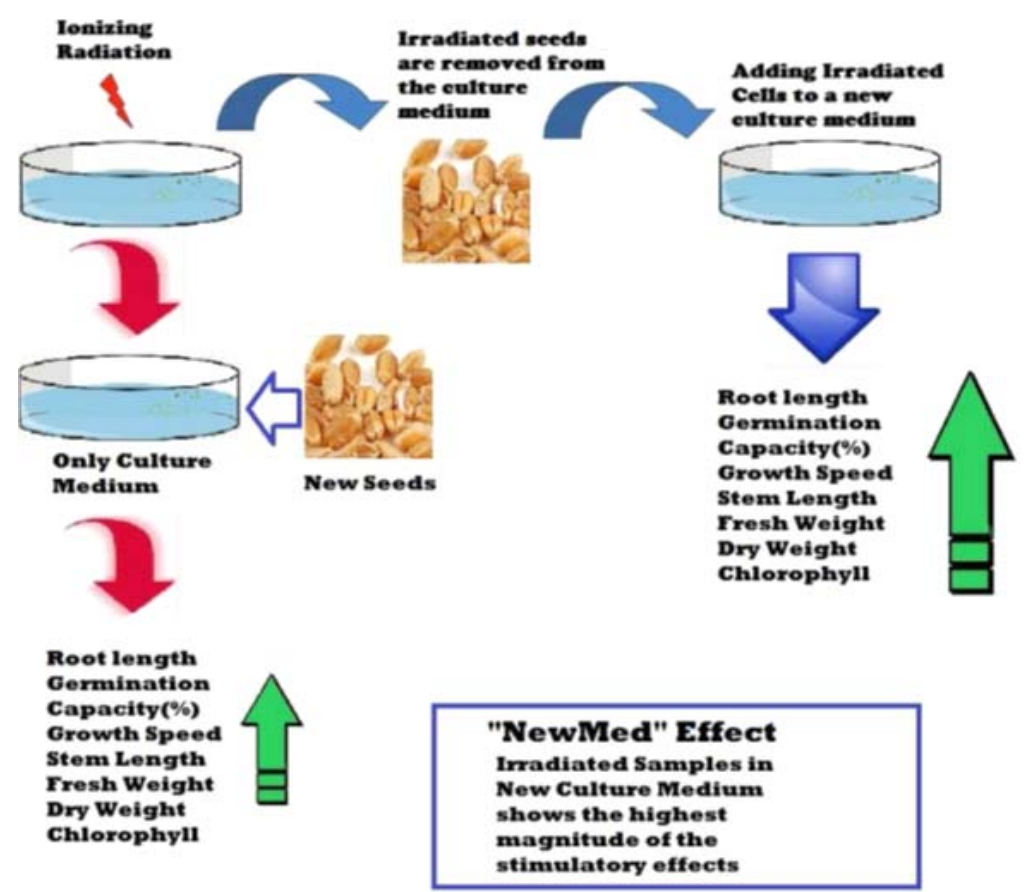

Fig. 11. The Graphical representation of the NewMed effect. When irradiated seeds were transferred to a new unirradiated culture medium, the biopositive effects of low doses of ionizing radiation were significantly amplified compared to those of irradiated seeds which were not transferred to a new un-irradiated culture medium

\section{DISCUSSION}

To the best of our knowledge this the first report on a phenomenon we called it "NewMed" effect. Furthermore, as far as we know, this is the 1 st report on the stimulatory bioeffects of exposure to very low doses of gamma radiation in Triticum aestivum. The findings of this study clearly showed that exposure of Triticum aestivum to very low levels of ionizing radiation in the range of a few $\mathrm{mSv}$ enhances important factors such as root length, stem length, germination capacity, germination speed, fresh weight and the chlorophyll content. Since a great proportion of the plant water uptake is taking place by root systems and one of the first responses of plants to water stress is the root elongation (Taiz L 2002), enhancement of this response through various factors, including irradiation can improve their resistance to drought. Further research in this field can open new horizons in global food production and potential applications of this phenomenon overcoming drought and food shortage. It is worth mentioning that a dangerous hunger crisis has been warned by the United Nations because the world grain reserves are so risky low that severe climate in the food-exporting countries could trigger such a global catastrophe. Although wheat (Triticum aestivum) is an important food source in the world, it fails to grow properly in many areas due to unfavorable climate and soil conditions. Our findings in this section of the study are generally in line with reports which indicated a broad spectrum of hormetic effects due to stressors such as ionizing and non-ionizing radiation, heat, caloric intake, and even exercise, on plants, fungi, bacteria, protozoa, and animals, including humans (Barceló and Poschenrieder 2002; Calabrese and Baldwin 2000; Feinendegen 2005; Garzon and Flores 2013; Hayes 2007; Hayes 2009; Ji et al. 2006; Mortazavi et al. 2014a; Mortazavi et al. 2012a; Mortazavi 2013a; Mortazavi 2013b; Mortazavi 2013c; Mortazavi 2014; Mortazavi et al. 2011; Mortazavi et al. 2013; Mortazavi et al. 2014b; Rattan 2008; Tlili et al. 2011). Feinendegen has previously reported that low doses in the mGy range cause a dual effect on cellular DNA; a low risk of DNA damage and the induction of an adaptive protection 
against DNA damages from many, mainly endogenous, sources. He has reported that exposure to doses in the range of background radiaton, the damage to DNA is orders of magnitude lower than that from endogenous sources (i.g. reactive oxygen species) (Feinendegen 2005).

As the most important finding in these studies, we discovered that the biopositive effects of low doses of ionizing radiation were significantly amplified compared to those of irradiated seeds which were not transferred to a new un-irradiated culture medium. We found that in Triticum aestivum transferring irradiated seeds to a new un-irradiated culture medium, significantly enhances root length, stem length, dry weight and the chlorophyll content. It should be noted that as an abiotic stress, electromagnetic radiation can induce oxidative processes in plant cells. Moreover, exposure to radiation in the presence of $\mathrm{O} 2$, induces the production of reactive oxygen species (ROS), such as superoxide, hydrogen peroxide and hydroxyl radical (Fan X. 2004). As plants are sessile and cannot escape the biotic and abiotic stresses, they depend on neatly developed signaling networks, including ROS signaling pathways, to regulate various developmental processes and responses to environmental stimuli (Heidarvand L 2010; Petrov VD 2012). It has been shown that the amount of ROS produced is related to the severity of stress; if the cells are exposed to heavy stress, large quantities of ROS will be produced as toxic byproducts of metabolism (Mittler R 2011; Potters G 2010; Thomas 2008). ROS have the ability to react with essential molecules, such as nucleic acids, proteins, lipids and carbohydrates, and alter their normal properties. In contrast, a weak stress induces low levels of ROS and these level of ROS can regulate various processes of plant growth and development through different signaling pathways, Such as calcium and protein kinase (MAPK) (Rathinasabapathi 2013). In the light of these events, we believe that in spite of this fact that exposure to low levels of ionizing radiation leads to stimulatory effects in irradiated seeds which leads to enhanced growth, agents released in the culture medium by irradiated seeds can limit the growth of these seeds. This concept can entirely explain the necessity of removing the irradiated medium and transferring the irradiated seeds to a new medium for obtaining better growth characteristics. As it has been shown that oxidative stress can be an important mediator of radiation induced bystander effect (Harada et al. 2008; Widel 2012), it can be hypothesized that oxidative stress induced by low doses of ionizing radiation has triggered the "NewMed effect" in irradiated seeds. Further studies are needed to investigate if this effect can be observed at cellular level. On the other hand, different aspects of this phenomenon and its potential applications should be further studied.

\section{ACKNOWLEDGEMENTS}

This study was supported by the Ionizing and Non-ionizing Radiation Protection Research Center (INIRPRC), Shiraz University of Medical Sciences (SUMS), Shiraz, Iran.

\section{REFERENCES}

1. Barceló J, and Poschenrieder C. Fast root growth responses, root exudates, and internal detoxification as clues to the mechanisms of aluminium toxicity and resistance: a review. Environmental and Experimental Botany 2002; 48(1):75-92.

2. Baskar R. Emerging role of radiation induced bystander effects: Cell communications and carcinogenesis. Genome Integrity 2010; 1(1):13.

3. Baskar R, Balajee AS, and Geard CR. Effects of low and high LET radiations on bystander human lung fibroblast cell survival. International journal of radiation biology 2007; 83(8):551-559.

4. Calabrese EJ, and Baldwin LA. Radiation hormesis: its historical foundations as a biological hypothesis. Human \& experimental toxicology 2000; 19(1): 41-75.

5. Fan X. TDWaSKJ. Changes in growth and antioxidant status of alfalfa sprouts during sprouting as affected by gamma irradiation of seeds. J Food Prot 2004; 67(3):561-566.

6. Feinendegen LE. Evidence for beneficial low level radiation effects and radiation hormesis. The British journal of radiology 2005; 78(925): 3-7.

7. Garzon CD, and Flores FJ. Hormesis: Biphasic Dose-Responses to Fungicides in Plant Pathogens and Their Potential Threat to Agriculture, 2013.

8. Ghiassi-Nejad M, Mortazavi S, Cameron J, Niroomand-Rad A, and Karam P. Very high background radiation areas of Ramsar, Iran: preliminary biological studies. Health Physics 
2002; 82(1): 87.

9. Hall EJ. The bystander effect. Health Phys 2003; 85(1): 31-35.

10. Han W, Chen S, Yu KN, and Wu L. Nitric oxide mediated DNA double strand breaks induced in proliferating bystander cells after alpha-particle irradiation. Mutat Res 2010; 684(1-2):81-89.

11. Hayes DP., Nutritional hormesis. European journal of clinical nutrition 2007; 61(2):147-159.

12. Hayes DP. Nutritional hormesis and aging. Doseresponse : a publication of International Hormesis Society 2009; 8(1): 10-15.

13. Heidarvand L MAR. What happens in plant molecular responses to cold stress? Acta Physiol Plant 2010; 32(3): 419-431.

14. Ji LL, Gomez-Cabrera MC, and Vina J. Exercise and hormesis: activation of cellular antioxidant signaling pathway. Annals of the New York Academy of Sciences 2006; 1067:425-435.

15. Melki MaDS. Studies the effects of low dose of gamma rays on the behaviour of chickpea under various conditions. Pak J Biol Sci 2008; 11:23262330.

16. Harada T, Kashino G, Suzuki K, Matsuda N, Kodama S, and Watanabe M. Different involvement of radical species in irradiated and bystander cells. International journal of radiation biology 2008; 84(10):809-814.

17. Melki MM, A., Effects of gamma rays irradiation on seed germination and growth of hard wheat. Environ chem leu 2010; 8: 307-310.

18. Mittler R VS, Suzuki N, Miller G,Tognetti VB, Vandepoele $\mathrm{K}$, et al. ROS signaling: the new wave? Trends Plant Sci 2011; 16: 300-309.

19. Mortazavi S, Ghiassi-Nejad M, and Rezaiean M. Cancer risk due to exposure to high levels of natural radon in the inhabitants of Ramsar, Iran. International Congress Series 2005a; 1276:436437.

20. Mortazavi SAR, Tavakkoli-Golpayegani A, Haghani M, and Mortazavi SMJ. Looking at the other side of the coin: The search for possible biopositive cognitive effects of the exposure to $900 \mathrm{MHz}$ GSM mobile phone radiofrequency radiation. Journal of Environmental Health Science and Engineering 2014a; 12(1).

21. Mortazavi SM, Rouintan MS, Taeb S, Dehghan N, Ghaffarpanah AA, Sadeghi Z, and Ghafouri F. Human short-term exposure to electromagnetic fields emitted by mobile phones decreases computer-assisted visual reaction time. Acta Neurol Belg 2012a; 112(2):171-175.

22. Mortazavi SMJ. Space radiobiology and the new era of induced radioresistance: Should traditional concepts be moved to science history museums? Technology and Health Care 2013a; 21(4):285-
289.

23. Mortazavi SMJ. Space research and EMFinduced adaptive responses. Journal of Medical Hypotheses and Ideas 2013b; 7(1):1-2.

24. Mortazavi SMJ. Window Theory in NonIonizing Radiation-Induced Adaptive Responses. Doseresponse : a publication of International Hormesis Society in press, 2013c.

25. Mortazavi SMJ. Is mobile phone radiofrequency radiation all bad? Journal of Medical Hypotheses and Ideas 2014; 8(1):42-43.

26. Mortazavi SMJ, Abbasi A, Asadi R, and Hemmati A., The need for considering social, economic, and psychological factors in warning the general public from the possible risks due to residing in HLNRAs. International Congress Series 2005b; 1276(0):440-441.

27. Mortazavi SMJ, and Karam PA. Apparent lack of radiation susceptibility among residents of the high background radiation area in Ramsar, Iran: can we relax our standards? Natural Radiation Environment 2005; 7:1141-1147.

28. Mortazavi SMJ, Mosleh-Shirazi MA, Tavassoli AR, Taheri M, Bagheri Z, Ghalandari R, Bonyadi S, Shafie M, and Haghani M. A comparative study on the increased radioresistance to lethal doses of gamma rays after exposure to microwave radiation and oral intake of flaxseed oil. Iranian Journal of Radiation Research 2011; 9(1):9-14.

29. Mortazavi SMJ, Mosleh-Shirazi MA, Tavassoli AR, Taheri M, Mehdizadeh AR, Namazi SAS, Jamali A, Ghalandari R, Bonyadi S, Shafie M et al. Increased Radioresistance to Lethal Doses of Gamma Rays in Mice and Rats after Exposure to Microwave Radiation Emitted by a GSM Mobile Phone Simulator. Dose-response : a publication of International Hormesis Society 2013; 11(2):281-292.

30. Mortazavi SMJ, Motamedifar M, Namdari G Taheri M, Mortazavi AR, and Shokrpour N. Nonlinear adaptive phenomena which decrease the risk of infection after pre-exposure to radiofrequency radiation. Dose-Response 2014b; 12(2):233-245.

31. Mortazavi SMJ, and Mozdarani H. Is it time to shed some light on the black box of health policies regarding the inhabitants of the high background radiation areas of Ramsar? Iranian Journal of Radiation Research 2012; 10(3-4):111-116.

32. Mortazavi SMJ, and Mozdarani H. Non-linear phenomena in biological findings of the residents of high background radiation areas of Ramsar. International Journal of Radiation Research 2013; 11(1): 3-9.

33. Mortazavi SMJ, Niroomand-Rad A, Mozdarani H, Roshan-Shomal P, Razavi-Toosi SMT, and 
Zarghani H., Short-term exposure to high levels of natural external gamma radiation does not induce survival adaptive response. Iranian Journal of Radiation Research 2012b; 10(34):165-170.

34. Mortazavi SMJ, Shabestani-Monfared A, Ghiassi-Nejad $M$, and Mozdarani $H$. Radioadaptive responses induced in lymphocytes of the inhabitants in Ramsar, Iran. International Congress Series 2005c; 1276(0): 201-203.

35. Petrov VD BF. Hydrogen peroxide-a central hub for information flow in plant cells. Cell Biology 2012; 14:1093-1206.

36. Potters G HN, Jansen MAK. The cellular redox state in plant stress biology-a charging concept. Plant Physiol Biochem 2010; 48:292-300.

37. Rathinasabapathi AKaB. Oxidative stress tolerance in plants. Plant Signaling \& Behavior 2013; 8(10). Rattan SI., Hormesis in aging.
Ageing research reviews 2008; 7(1):63-78.

38. Ryan L, Smith R, Seymour C, and Mothersill C. Dilution of Irradiated Cell Conditioned Medium and the Bystander Effect. Radiation research 2008; 169:188 - 196.

39. Taiz L ZE. Plant physiology. Sunderland: Sinauer Associates INC, 2002.

40. Thomas DD, Ridnour, L.A. , Isenberg, J.S., Flores-Santana,W. ,Switzer,C.H. ,Donzelli,S.,et al. The chemical biology of nitricoxide: implicationsin cellular signaling. Free Radic Biol Med 2008; 45:18-31.

41. Tlili A, Montuelle B, Berard A, and Bouchez A. Impact of chronic and acute pesticide exposures on periphyton communities. The Science of the total environment 2011; 409(11):2012-2013.

42. Widel M. Bystander effect induced by UV radiation; why should we be interested? Postepy higienyi medycyny doswiadczalnej (Online) 2012; 66: 828-837. 